\title{
Heart Rate Variability is an Independent Predictor of Lymph Node Metastasis in Patients with Cervical Cancer
}

\author{
Jingfeng Wang $\mathbb{D}^{1,2, *}$ \\ Jian $\mathrm{Liu}^{3, *}$ \\ Longfei $\mathrm{Gao}^{3}$ \\ Guangqiao $\mathrm{Li}^{1,2}$ \\ Yilin Sun ${ }^{3}$ \\ Bo Shi ${ }^{1,2}$
}

'School of Medical Imaging, Bengbu Medical College, Bengbu, Anhui, 233030, People's Republic of China; ${ }^{2}$ Anhui Key Laboratory of Computational Medicine and Intelligent Health, Bengbu Medical College, Bengbu, Anhui, 233030, People's Republic of China; ${ }^{3}$ Department of Gynecologic Oncology, First Affiliated Hospital, Bengbu Medical College, Bengbu, Anhui, 233004, People's Republic of China

*These authors contributed equally to this work
Correspondence: Bo Shi

School of Medical Imaging, Bengbu

Medical College, Bengbu, Anhui, People's

Republic of China

Tel +86-18955203003

Email shibo@bbmc.edu.cn
Purpose: Heart rate variability (HRV) has been reported as a useful biomarker for prognostic factors in a variety of cancers. The purpose of this study was to explore the predictive value of preoperative HRV for lymph node metastasis (LNM) in patients with cervical cancer (CC).

Patients and Methods: A total of 77 patients with CC were included, including 18 LNM and 59 non-LNM patients. A five-minute resting electrocardiogram (ECG) was collected before surgery for the analysis of HRV time domain, frequency domain and Poincaré plot parameters (ie, SDNN, RMSSD, LF, HF, LF/HF, SD1, SD2 and SD2/SD1). Student's $t$-tests and logistic regression were performed to determine the relationship between HRV and LNM.

Results: The LNM group had significantly lower SDNN, LF, and SD2 than the non-LNM group (all $p<0.05$; all Cohen's $d>0.5$ ). Binary logistic regression analysis indicated that SDNN, LF and SD2 were still significantly associated with LNM. Specifically, for each $1 \mathrm{~ms}$ decrease in SDNN and SD2 and each 1 logarithmic unit decrease in $\ln (\mathrm{LF})$, the odds of LNM increased by $12 \%, 9 \%$, and $86 \%$, respectively (all $p<0.05$ ).

Conclusion: These findings suggest an association between HRV and CC LNM, and HRV could be a potential noninvasive biomarker for the prediction of LNM in CC patients.

Keywords: cervical cancer, heart rate variability, lymph node metastasis, autonomic nervous system

\section{Introduction}

Cervical cancer (CC) is a common malignant cancer in women, with an estimated global incidence of approximately 500,000 new cases and approximately 250,000 deaths per year. ${ }^{1}$ Annual new cases of CC in China account for approximately $30 \%$ of cases worldwide. ${ }^{2}$ At present, more than $80 \%$ of CC patients in China are mainly treated with surgery, and the 5 -year survival rate exceeds $65 \%{ }^{2,3}$ Lymph node metastasis (LNM) has long been considered an important risk factor and the leading cause of death from CC. ${ }^{4}$ Studies have shown that the 5-year survival rate of CC patients declines dramatically from $95 \%$ in patients without LNM to $51 \%$ in patients with LNM. ${ }^{5}$ Therefore, the occurrence of LNM in CC patients is a key factor affecting treatment decisions, especially preoperative adjuvant chemotherapy. ${ }^{3}$ The early diagnosis of LNM is of great significance for guiding the personalized treatment of $\mathrm{CC}$ patients and improving the prognosis of patients.

Imaging examinations, such as computed tomography (CT), endoscopic ultrasound (EUS), magnetic resonance imaging (MRI) and positron emission tomography-computed tomography (PET-CT), are common methods for the preoperative 
evaluation of LNM. However, the diagnostic model of imaging is based on size and morphology, and the sensitivity and specificity of scanning for smaller lymph nodes are still low. ${ }^{6,7}$ Some novel molecular biomarkers, including gene expression profiles, transcription factors and protein expression, have also been used for LNM prediction in recent years. ${ }^{8-12}$ However, the high cost and complicated technology restrict their clinical application. Hematological biomarkers (eg, tumor markers and inflammatory markers) are also widely used to quantify risk factors for LNM in various cancers, including CC. ${ }^{13,14}$ Tumor markers, such as squamous cell carcinoma antigen (SCC-Ag) and cancer antigen 125 (CA125), and inflammatory markers, including the neutrophil-to-lymphocyte ratio (NLR) and platelet-to-lymphocyte ratio (PLR), are predictors of CC LNM. ${ }^{15,16}$ Nevertheless, these indicators still have deficiencies in accuracy and consistency, and the best blood biomarker for predicting prognosis is also controversial. As a result, it is still urgent to establish effective methods for assessing LNM before surgery.

Autonomic nervous system (ANS) function is an important component involved in cancer etiology and is related to tumor occurrence, progression, immune response, vascular infiltration, and lymphatic metastasis. ${ }^{17-19}$ ANS characterized by heart rate variability (HRV) has been extensively studied in the prognostic assessment of cancer. ${ }^{20,21}$ There is growing evidence that preoperative HRV is closely associated with the pathology of a variety of malignant tumors. For example, Bijoor et al analyzed the HRV time domain parameters of patients with different stages of malignant tumors (head and neck cancer, gastrointestinal cancer, and gynecological cancer) and healthy people. The results showed that the root mean square of successive interval differences (RMSSD) in tumor patients was significantly lower than that in healthy people, and the RMSSD in patients with advanced tumors (stage III and IV) was significantly lower than that in patients with early-stage disease (stage I and II). ${ }^{22}$ Bettermann et al compared the difference in the HRV nonlinear index in breast cancer (BC) patients with and without metastasis and found that metastatic patients had a significantly lower approximate entropy than nonmetastatic patients. The results indicated that the variability, complexity and irregularity of heart rate dynamics in metastatic patients decreased significantly. ${ }^{23}$ Arab et al also studied the relationship between tumor stage and time and frequency domain HRV parameters in BC patients. The results showed that the later the tumor stage is, the more significant the decrease in time domain parameters (standard deviation of all normal-to-normal intervals (SDNN) and RMSSD). ${ }^{24}$ In addition, $\mathrm{Hu}$ et al reported that HRV decreased with tumor progression in patients with gastric cancer (GC) and was related to clinical stage, tumor size, depth of invasion, lymphatic metastasis, and distant metastasis. ${ }^{25}$

However, it has yet to be verified whether HRV is linked to LNM in CC patients. The purpose of this study was to compare the difference in preoperative HRV between CC patients with and without LNM. We hypothesized that the LNM group has a lower HRV than the non-LNM group. Once confirmed, HRV may play an important role in the early prediction and prognosis of LNM in CC patients.

\section{Materials and Methods Subjects}

The subjects were 81 patients with $\mathrm{CC}$ admitted to the Department of Gynecology and Oncology, the First Affiliated Hospital of Bengbu Medical College (Anhui, China), from November 2020 to April 2021. The inclusion criteria were as follows: (1) CC diagnosed by pathological examination; (2) new patients, without surgery, radiotherapy, or chemotherapy; and (3) met the indications for CC radical surgery and treated with extensive hysterectomy and pelvic lymphadenectomy. The exclusion criteria were as follows: (1) patients during pregnancy; (2) patients with primary diseases of vital organs (such as the heart, liver, and kidneys); and (3) patients with other tumors. This study was approved by the Clinical Medical Research Ethics Committee of The First Affiliated Hospital of Bengbu Medical College (Bengbu, Anhui, China) (registration number: 2021KY010). The experiments were conducted in strict accordance with the ethical standards set forth in the 1964 Declaration of Helsinki and its later amendments. All patients were informed of the details of the study, procedures, risks and potential adverse effects of the experiment and signed an informed consent form.

\section{Data Collection}

A single-lead electrocardiogram (ECG) recorder (version 2.8.0, Healink-R211B, Healink Ltd., Bengbu, China) was used 1 day prior to surgery to collect ECG data $(5 \mathrm{~min})$ from CC patients while in a supine position. The sampling rate of the ECG recorder was $400 \mathrm{~Hz}$, and the signal bandwidth was $0.6-40 \mathrm{~Hz}$. During the test, the subjects were asked to remain quiet and breathe smoothly. A V6 
lead was employed, and the measuring electrodes were $\mathrm{Ag} / \mathrm{AgCl}$ disposable gel ECG electrode sheets (Junkang Ltd., Shanghai, China). The test environment was kept quiet, and the temperature was maintained at $23 \pm 1{ }^{\circ} \mathrm{C}$.

In all patients, the dissected pelvic lymph nodes included the common iliac, external iliac, internal iliac, and obturator fossa nodes on both sides. Then, the lymph nodes were marked and sent to the Pathology Department for examination. Each lymph node was sectioned and examined for LNM by routine hematoxylin and eosin staining. Based on the examination results, the patients were divided into two groups: the LNM group $(\mathrm{n}=18)$ and the non-LNM group $(n=59)$.

\section{HRV Analysis}

The Pan-Tompkins algorithm was used to extract the R-to$\mathrm{R}$ interval (RRI) time series. ${ }^{26}$ The artifacts caused by the extraction algorithm, interference and ectopic heartbeat were automatically corrected by a time-varying threshold algorithm. $^{27}$ Then, HRV time domain, frequency domain and nonlinear analyses were performed.

The time domain parameters included the SDNN and RMSSD. $^{28}$

The frequency domain parameters included the lowfrequency (LF; $0.04-0.15 \mathrm{~Hz}$ ) power, the high-frequency (HF; $0.15-0.40 \mathrm{~Hz}$ ) power, and the ratio of LF power to HF power (LF/HF). Prior to frequency domain analysis, the RRI time series was resampled at $4 \mathrm{~Hz}$ using cubic spline interpolation. $^{29}$ The fast Fourier transform (FFT) with Welch's periodogram method (with $150 \mathrm{~s}$ window width and $50 \%$ overlapping window) was applied to estimate the power spectral density of the RRI time series. ${ }^{30}$ The spectrum was obtained by averaging the spectra of these overlapping segments to decrease the variance of the FFT spectrum.

Poincaré scatter plots are a commonly used method in HRV nonlinear analysis; they graphically represent the correlation between successive RRIs. ${ }^{31}$ By fitting the ellipse into the plot, three important indexes are obtained, namely, the length of the semi-major axis of the ellipse (SD2), the length of the semi-minor axis of the ellipse (SD1), and the ratio of the two (SD2/SD1).

The respiratory rate $(\mathrm{RR})$ has an important influence on HRV. The RR located in the HF band $(0.15-0.40 \mathrm{~Hz})$ of HRV is the basis of the true response of LF- and HFrelated indexes to autonomic nerves. Conversely, when the RR is in the LF band or higher than the HF band, it will lead to a misunderstanding of sympathetic and vagal nerve activity. ${ }^{32}$ Therefore, the separation of respiratory influences from HRV can better evaluate the activity of the ANS. RR extraction can be achieved by the ECG-derived respiration method, and we named this variable EDRR. ${ }^{33}$

The analyses of the above processing steps were performed with Kubios HRV Premium software (version 3.1.0, https://www.kubios.com,KubiosOy,Kuopio, Finland). ${ }^{34}$

\section{Statistical Analysis}

All data were first subjected to the Shapiro-Wilk normality test. RMSSD, LF, HF, and LF/HF values were natural logarithmically transformed prior to further analysis, as they were right-skewed. The chi-square test and unpaired Student's $t$-tests were used to compare characteristics between the two groups. HRV parameters were compared by using unpaired Student's $t$-tests. An effect size measured by Cohen's $d$ statistic was also reported. The effect size was considered small if $d<0.5 .^{35}$ A logistic regression model was built to estimate the odds ratio (OR) value while adjusting for age, BMI, EDRR, mean heart rate (HR), hypertension, diabetes, and menopausal status. To avoid collinearity, the HRV parameters were each included in a separate regression model as a predictor. The Benjamini-Hochberg procedure was used to control for the false discovery rate (FDR) across multiple comparisons. Statistical significance was considered when FDRcorrected $p<0.05$.

\section{Results}

The ECG data of a total of 81 CC patients were collected, with several patients excluded due to poor ECG quality (n $=1)$, ectopic heartbeats $>10 \%$ of all beats $(n=1)$ or abnormal data extremes $(\mathrm{n}=2)$. The remaining $77 \mathrm{CC}$ patients (age: $52.1 \pm 10.0$ years; BMI: $24.4 \pm 3.0 \mathrm{~kg} / \mathrm{m}^{2}$ ) were included in this study. Table 1 summarizes the demographics and HRV parameters of all participants. There were no significant differences in characteristics, including age, BMI, EDRR, mean HR, pathological type, tumor size and invasion depth, between the two groups (Table 2).

Differences in HRV parameters (time domain, frequency domain and nonlinear parameters) between the two groups are shown in Figure 1. The effect sizes of the differences are shown in Figure 2. The results showed that SDNN (Figure 1, $p<0.0001$, Figure 2, $d>0.5$ ), LF (Figure 1, $p=0.003$, Figure 2, $d>0.8$ ), and SD2 (Figure 1, $p<0.0001$, Figure 2, $d>0.5$ ) were significantly decreased in the LNM group compared with the non-LNM group. 
Table I Demographics and HRV of Cervical Cancer Patients.

\begin{tabular}{|l|l|}
\hline Variables & Values \\
\hline N (Female) & 77 \\
Age (years) & $52.1 \pm 10.0$ \\
BMI (kg/m²) & $24.4 \pm 3.0$ \\
EDRR (Hz) & $0.3 \pm 0.1$ \\
Mean HR (bpm) & $72.8 \pm 8.8$ \\
Hypertension (yes/no) & $5 / 72$ \\
Diabetes (yes/no) & $3 / 74$ \\
Menopausal status (yes/no) & $47 / 30$ \\
Lymph node metastasis (yes/no) & $18 / 59$ \\
SDNN (ms) & $27.6 \pm 11.1$ \\
RMSSD (ms) & $2.8 \pm 0.6$ \\
LF (ms ${ }^{2}$ ) & $4.6 \pm 0.8$ \\
HF (ms ${ }^{2}$ ) & $4.6 \pm 1.1$ \\
LF/HF & $0.009 \pm 1.034$ \\
SDI (ms) & $13.0 \pm 7.6$ \\
SD2 (ms) & $36.5 \pm 14.6$ \\
SD2/SD I & $3.339 \pm 1.445$ \\
\hline
\end{tabular}

Note: Values are expressed as the number of patients or mean \pm standard deviation. Abbreviations: $\mathrm{N}$, number of individuals; BMI, body mass index; EDRR, ECGderived respiration rate; bpm, beats per minute; HR, heart rate; SDNN, standard deviation of all normal-to-normal intervals; RMSSD, root mean square of successive interval differences; LF, low-frequency power; HF, high-frequency power; LF/HF, ratio of low-frequency power to high-frequency power; SD, standard deviation.

Table 2 Characteristics of the Patients According to LNM Status.

\begin{tabular}{|l|l|l|l|}
\hline Variables & Non-LNM (n=59) & LNM (n=18) & $\mathbf{p}$ \\
\hline Age (years) & $51.1 \pm 9.7$ & $55.2 \pm 10.2$ & 0.130 \\
BMI $\left(\mathrm{kg} / \mathrm{m}^{2}\right)$ & $24.4 \pm 3.0$ & $24.6 \pm 2.8$ & 0.745 \\
Mean HR (bpm) & $72.6 \pm 8.9$ & $73.6 \pm 8.6$ & 0.693 \\
EDRR (Hz) & $0.271 \pm 0.055$ & $0.275 \pm 0.069$ & 0.764 \\
\hline Pathological type & & & \\
SCC & 49 & 10 & 0.550 \\
Non-SCC & 16 & 2 & \\
\hline Tumor size $(\mathrm{cm})$ & & & \\
$<4 \mathrm{~cm}$ & 43 & 10 & 0.165 \\
$\geq 4 \mathrm{~cm}$ & 16 & 8 & \\
\hline Invasion depth & & & \\
$<2 / 3$ & 36 & 11 & \\
$\geq 2 / 3$ & 23 & 7 & \\
\hline
\end{tabular}

Note: Values are expressed as the number of patients or mean \pm standard deviation. Abbreviations: BMI, body mass index; EDRR, ECG-derived respiration rate; HR, heart rate; bpm, beats per minute; SCC, squamous cell carcinoma.

Binary logistic regression analysis indicated that SDNN, LF and SD2 were still significantly associated with LNM. Specifically, for each $1 \mathrm{~ms}$ decrease in SDNN and SD2 and each 1 logarithmic unit decrease in $\ln (\mathrm{LF})$, the odds of LNM increased by $12 \%, 9 \%$, and $86 \%$, respectively [all FDR-corrected $p<0.05$; Table 3]. The association was independent of potential confounding factors, including age, BMI, EDRR, mean HR, hypertension, diabetes, and menopausal status.

\section{Discussion}

In this study, we compared the HRV time domain, frequency domain, and Poincaré scatter plot-based nonlinear parameters of $\mathrm{CC}$ patients with and without LNM. As expected, the results showed that the HRV parameters (SDNN, LF and SD2) of the LNM group were significantly decreased compared with those of the non-LNM group. Importantly, this link was not associated with confounding factors such as age, BMI, EDRR, resting $\mathrm{HR}$, hypertension, diabetes or menopausal status.

In previous studies, some tumor pathological factors, including clinical stage, tumor size, lymphatic vascular space invasion (LVSI), depth of stromal invasion (DSI) and parametrial involvement (PI), have been reported as predictors of LNM in CC. ${ }^{36-40}$ For example, Fagotti et al found that $\mathrm{CC}$ patients with tumor diameter $\geq 20 \mathrm{~mm}$ had a higher risk of LNM. ${ }^{37}$ Gulseren et al demonstrated that LVSI $(\mathrm{OR}=11.3, p<0.001)$ and tumor size $\geq 2 \mathrm{~cm}(\mathrm{OR}=$ $3.2, p=0.005)$ were independent predictors of pelvic lymph node involvement. ${ }^{38}$ Nanthamongkolkul et al evaluated 496 patients with early cervical cancer (stages IA2IB1) and showed that DSI $(p=0.010)$ and PI $(p=0.005)$ were significantly and independently associated with LNM. ${ }^{40}$ However, these predictors are evident only after surgery or with the help of imaging examinations. Clinical studies have demonstrated that HRV is a potential noninvasive diagnostic tool for autonomic dysfunction and is widely used for the evaluation of prognosis in cancer patients. $^{20,21}$

SDNN and SD2 have been shown to be highly correlated in computational methods and research outcomes, both reflecting the overall variability and relating to the combination of sympathetic and vagal functions. ${ }^{41,42}$ Based on the complexity theory, in healthy bodies, the two branches of the ANS (ie, the sympathetic nerve and the vagus nerve) interact with each other and are in a state of dynamic equilibrium. ${ }^{43}$ This physiological selfregulation (ie, autonomic balance) equips the body with strong stability and the ability to adapt to different environments. The decrease in overall HRV in cancer patients indicates the impairment of complexity, stability and adaptability, as well as the loss of autonomic nerve 

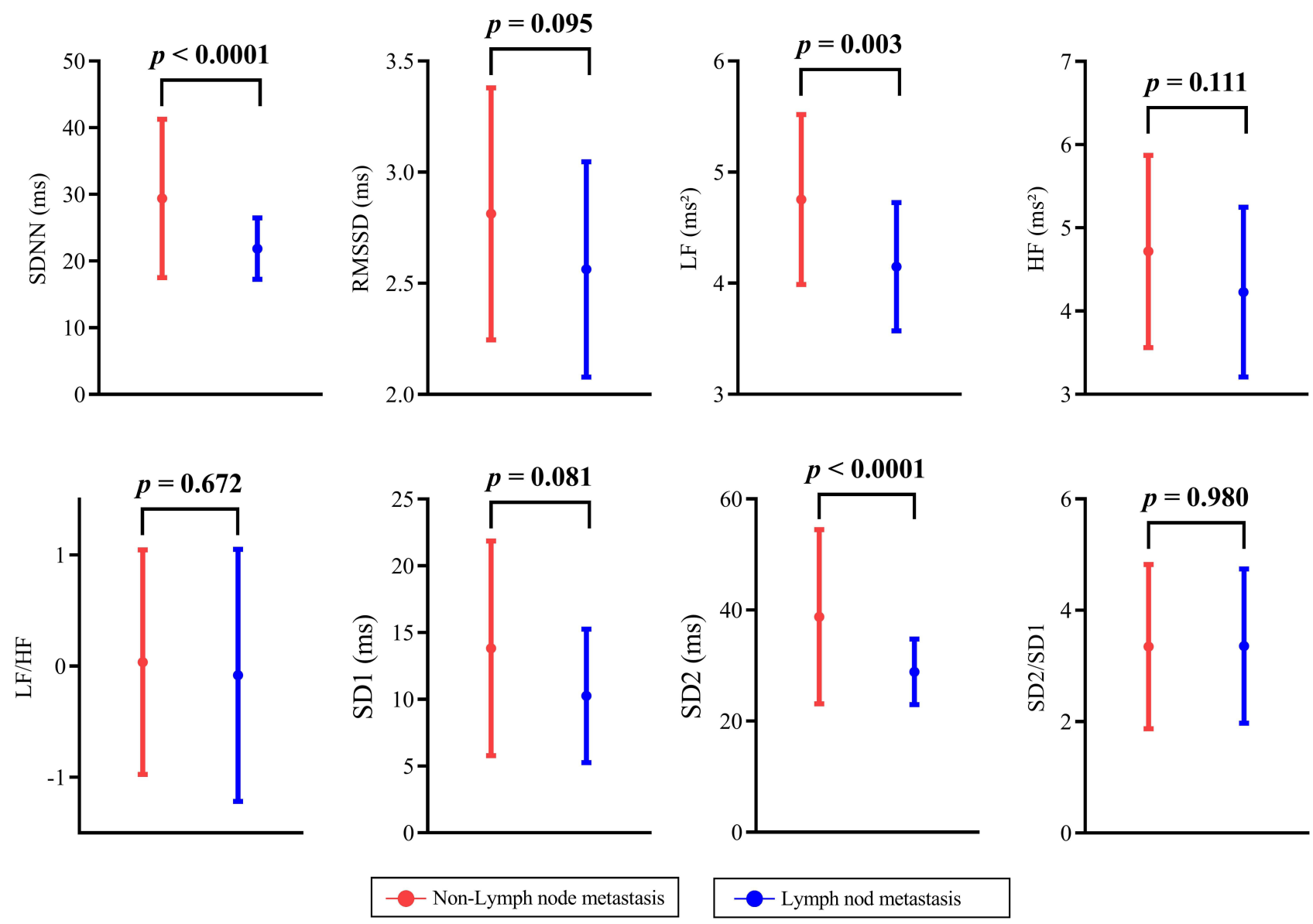

Figure I Group differences in HRV parameters between non-LNM and LNM.

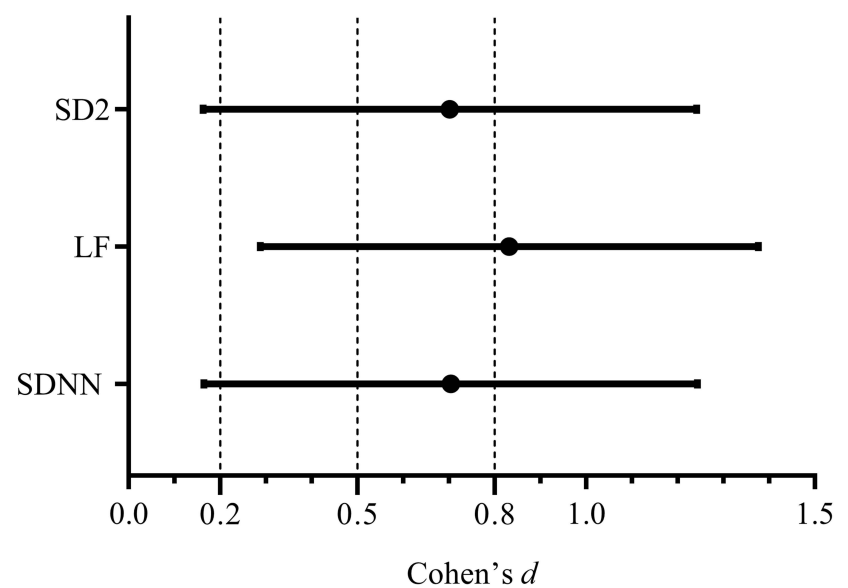

Figure 2 Effect size of differences in SDNN, LF and SD2 between the non-LNM and LNM groups.

balance, and predicts poor survival and prognosis in patients. ${ }^{4-46}$ In CC patients, the LNM group had lower SDNN and SD2, which is consistent with the findings of previous studies showing that tumor progression leads to a decrease in HRV.
Catecholamines released from the sympathetic nervous system (SNS) can activate $\beta$-adrenergic receptors on tumor cells, which regulate gene expression by activating multiple intracellular signal transduction pathways, thereby enhancing tumor angiogenesis and promoting tumor invasion and metastasis. $^{47}$ In previous studies, LF was related to SNS activity. $^{28}$ For example, Pagani et al found that sympathetic nerve activation can result in increased LF in dogs, and bilateral sympathetic stellectomy can lead to decreased LF. $^{48}$ If explained from this perspective, sympathetic activity in LNM patients will manifest as an increase in LF, which is clearly contrary to the findings of this study. In fact, other researchers have not observed that sympathetic activation, such as exercise or adrenergic agonists, can cause an increase in LF. ${ }^{49,50}$ Consequently, LF may not necessarily represent only sympathetic activity. Currently, the interpretation of LF remains controversial. In recent reports, LF was found to predominantly reflect the autonomic nerve activity regulated by the baroreceptor reflex. ${ }^{51,52}$ The vagus nerve is the main channel through which afferent nerve signals (including 
Table 3 Results from Logistic Regression Models (Adjusted for Age, BMI, EDRR, Resting Mean HR, Hypertension, Diabetes, and Menopausal Status).

\begin{tabular}{|l|l|l|l|l|l|}
\hline Variables & OR $(\mathbf{9 5} \% \mathbf{~ C l )}$ & $\mathbf{p}$ & $\mathbf{R a n k}$ & $\mathbf{Q}$ & $\mathbf{p}<\mathbf{Q} ?$ \\
\hline LF & $0.238(0.133,0.707)$ & 0.007 & 1 & 0.0167 & TRUE \\
SDNN & $0.880(0.789,0.980)$ & 0.020 & 2 & 0.0333 & TRUE \\
SD2 & $0.908(0.837,0.985)$ & 0.020 & 3 & 0.05 & TRUE \\
\hline
\end{tabular}

Notes: Q: the Benjamini-Hochberg critical value [= (rank/number of tests) $\times$ FDR]. TRUE for $P<Q$ indicates statistical significance after correcting for the FDR.

Abbreviations: OR, odds ratio; $\mathrm{Cl}$, confidence interval; SDNN, standard deviation of all normal-to-normal intervals; LF, low-frequency power; SD, standard deviation.

baroreceptor reflex signals) from the heart and other visceral organs are transmitted to the brain. ${ }^{53}$ Therefore, the decrease in LF may indicate vagus nerve damage and changes in autonomic nerve function. Lin et al analyzed HRV in metastatic and nonmetastatic cancer patients (head and neck cancer, lung cancer and BC). Similar to our results, the authors found that LF and total power (TP) were significantly lower in metastatic patients than in nonmetastatic patients. ${ }^{54}$ Furthermore, due to the complexity of LF, the complex nonlinear interaction between sympathetic and vagus nerve activity, and confounding by respiration and resting HR, it is difficult to discern the physiological basis of LF/HF. ${ }^{55}$ Therefore, LF/HF cannot accurately evaluate the sympathetic-vagal balance. This may also be the reason why we did not find a difference in LF/HF between the two groups in our study.

The ANS, particularly the vagus nerve, is closely related to the tumorigenesis and development of tumors. ${ }^{18}$ The vagus nerve can modulate and decelerate tumor progression by inhibiting oxidative stress, inflammation and excessive sympathetic activity. ${ }^{56-58}$ The possible causal relationship between vagus nerve activity and reduced tumor progression has been confirmed in human and animal studies. For instance, Erin et al showed that tumor-bearing animals with vagotomy had more metastases, while anti-inflammatory drugs (CNI-1493) that activate vagus nerves can reduce metastasis in tumor-bearing mice. ${ }^{59,60}$ In patients with multiple types of cancers, such as BC, GC, liver cancer and pancreatic cancer, low vagal nerve activity measured by HRV significantly predicted shorter survival, higher tumor markers and worse prognosis. ${ }^{61-64}$ Therefore, the difference in HRV between the LNM and non-LNM groups could be caused by different vagus activities. RMSSD, HF and SD1 are all used to evaluate the modulation of the vagus nerve. ${ }^{42}$ However, in our study, there were no significant differences in RMSSD, HF or SD1 between the two groups. The possible reasons are as follows. On the one hand, age, BMI, EDRR, mean HR, pathological type, tumor size and invasion depth were not significantly different between the LNM group and the non-LNM group. The two groups showed high homogeneity. On the other hand, many factors, such as disease duration, cognitive performance, mental state and medication, will affect the measurement of HRV, especially measurements reflecting short-term variability and cardiac vagus modulation (RMSSD, HF and SD1). The predictive ability of these HRV parameters may be weakened or even absorbed by the above clinical risk factors. In contrast, nonlinear analysis is more sensitive to subtle changes in cardiovascular system dynamics and can reflect the complexity and nonlinear characteristics of autonomic nervous activity in the human heart. It is a promising tool for evaluating the changes in HRV in different physiological and pathological states. ${ }^{65-67}$

CC LNM is a complex process involving the interaction of multiple factors, such as immune function, inflammatory response and tumor microenvironment (TME). Impaired immune cell function, abnormal expression of immune factors and dysregulation of the immune system are important causes of CC LNM. ${ }^{68}$ Furthermore, regulatory $\mathrm{T}$ cells (Tregs) can inhibit the activity of antitumor cytotoxic $\mathrm{T}$ cells, induce immune tolerance to tumors, and promote tumor cell metastasis. ${ }^{69}$ Inflammatory cytokines (IL-1, IL-6, IL-8 and tumor necrosis factor- $\alpha$ ) and the TME also play an important role in tumor metastasis. ${ }^{70,71}$ Matrix metalloproteinases (MMPs) in the TME can degrade various protein components of the extracellular matrix (ECM), rebuild the ECM, destroy the tissue barrier, and mediate the metastasis of tumor cells. ${ }^{72}$ ANS can influence tumor LNM by modulating these factors. Specifically, the sympathetic nerve can inhibit antitumor immunity, induce tumor-promoting inflammation, potentiate angiogenesis or lymphangiogenesis in tumor tissue, and enhance the activity of MMPs, thus promoting tumor LNM. $^{73-76}$ The vagus nerve exerts a suppressive effect on LNM by inhibiting the activity of Tregs, enhancing the 
efficiency of antitumor cytotoxic T cells and the cholinergic anti-inflammatory pathway. ${ }^{77-79}$ Based on the above discussion, regarding the physiological and pathological mechanisms, patients with LNM will have autonomic imbalance and decreased vagus activity and will present with a decrease in HRV. In this study, a negative correlation between HRV and LNM was further confirmed, suggesting that vagus nerve damage is closely related to LNM and cancer progression, but its specific mechanism still needs in-depth research.

In the $\mathrm{CC}$ International Federation of Gynecology and Obstetrics (FIGO) staging system, LNM is an important indicator when evaluating the prognosis of CC. Our study found that lower HRV might potentially indicate LNM and increased severity in $\mathrm{CC}$ patients. Therefore, during the treatment of $\mathrm{CC}$, whether HRV can be activated by biofeedback (breathing, exercise, music, etc.) to improve the prognosis of patients with $\mathrm{CC}$ is worth investigating in future studies. $^{80-82}$

In recent years, radiomics methods based on artificial intelligence technology have been widely studied and applied in the prediction and prognosis of cancer. Radiomics extracts high-dimensional imaging features from medical images and can be used to predict LNM in many types of cancers, such as $\mathrm{CC}, \mathrm{BC}$ and colon cancer. ${ }^{83-85}$ If HRV parameters can be introduced into a radiomics model as a characteristic parameter, it is possible to achieve better prediction results, which is worthy of further exploration.

\section{Conclusion}

In conclusion, CC patients with LNM had lower HRV parameters (SDNN, LF and SD2) than those without LNM. The results of this study suggest that HRV could be a potential noninvasive biomarker for the prediction of LNM in CC patients. Combined with deep learning technology, HRV is expected to become a potential tool to guide the personalized treatment of $\mathrm{CC}$ patients. However, several limitations also existed in this study. Firstly, the sample size is relatively small and a larger sample size is required. Secondly, the effects of treatment, nutrition, psychology factors on HRV are not considered. Finally, the cross-sectional nature of the current study limits our ability to predict the long-term results of HRV parameters. The above questions deserve further study after expanding the sample size and considering more confounding factors.

\section{Acknowledgments}

This research was funded by the " 512 " Outstanding Talents Fostering Project of Bengbu Medical College (grant number BY51201312), and the Scientific Research Innovation Project of Bengbu Medical College (grant number BYKC201905).

\section{Disclosure}

A direct family member of B.S. owns stock in HeaLink Ltd., Bengbu, China. Other authors declare that there are no conflicts of interest.

\section{References}

1. Siegel RL, Miller KD, Jemal A. Cancer statistics, 2020. CA Cancer J Clin. 2020;70(1):7-30. doi:10.3322/caac.21590

2. Chen W, Zheng R, Baade PD, et al. Cancer statistics in China, 2015. CA Cancer J Clin. 2016;66(2):115-132. doi:10.3322/caac.21338

3. Marth C, Landoni F, Mahner S, McCormack M, Gonzalez-Martin A, Colombo N; ESMO Guidelines Committee. Cervical cancer: ESMO clinical practice guidelines for diagnosis, treatment and follow-up. Ann Oncol. 2017;28(suppl 4):iv72-iv83. doi:10.1093/annonc/ $\mathrm{mdx} 220$

4. Du R, Li L, Ma S, Tan X, Zhong S, Wu M. Lymph nodes metastasis in cervical cancer: incidences, risk factors, consequences and imaging evaluations. Asia Pac J Clin Oncol. 2018;14(5):e380-e385. doi:10.1111/ajco.12997

5. Gien LT, Covens A. Lymph node assessment in cervical cancer: prognostic and therapeutic implications. J Surg Oncol. 2009;99 (4):242-247. doi:10.1002/jso.21199

6. Williams AD, Cousins C, Soutter WP, et al. Detection of pelvic lymph node metastases in gynecologic malignancy: a comparison of $\mathrm{CT}$, MR imaging, and positron emission tomography. AJR Am J Roentgenol. 2001;177(2):343-348. doi:10.2214/ajr.177.2.1770343

7. Choi HJ, Ju W, Myung SK, Kim Y. Diagnostic performance of computer tomography, magnetic resonance imaging, and positron emission tomography or positron emission tomography/computer tomography for detection of metastatic lymph nodes in patients with cervical cancer: meta-analysis. Cancer Sci. 2010;101 (6):1471-1479. doi:10.1111/j.1349-7006.2010.01532.x

8. Kim TJ, Choi JJ, Kim WY, et al. Gene expression profiling for the prediction of lymph node metastasis in patients with cervical cancer. Cancer Sci. 2008;99(1):31-38. doi:10.1111/j.13497006.2007.00652.x

9. Huang L, Lin JX, Yu YH, Zhang MY, Wang HY, Zheng M. Downregulation of six microRNAs is associated with advanced stage, lymph node metastasis and poor prognosis in small cell carcinoma of the cervix. PLoS One. 2012;7(3):e33762. doi:10.1371/journal.pone. 0033762

10. Liu P, Zhang C, Liao Y, et al. High expression of PTPRM predicts poor prognosis and promotes tumor growth and lymph node metastasis in cervical cancer. Cell Death Dis. 2020;11(8):687. doi:10.1038/ s41419-020-02826-x

11. Chen J, Yao D, Li Y, et al. Serum microRNA expression levels can predict lymph node metastasis in patients with early-stage cervical squamous cell carcinoma. Int J Mol Med. 2013;32(3):557-567. doi:10.3892/ijmm.2013.1424

12. Wang W, Jia HL, Huang JM, et al. Identification of biomarkers for lymph node metastasis in early-stage cervical cancer by tissue-based proteomics. Br J Cancer. 2014;110(7):1748-1758. doi:10.1038/ bjc. 2014.92 
13. Kotowicz B, Fuksiewicz M, Kowalska M, Jonska-Gmyrek J, Bidzinski M, Kaminska J. The value of tumor marker and cytokine analysis for the assessment of regional lymph node status in cervical cancer patients. Int J Gynecol Cancer. 2008;18(6):1279-1284. doi:10.1111/j.1525-1438.2007.01176.x

14. Zhang LX, Wei ZJ, Xu AM, Zang JH. Can the neutrophil-lymphocyte ratio and platelet-lymphocyte ratio be beneficial in predicting lymph node metastasis and promising prognostic markers of gastric cancer patients? Tumor maker retrospective study. Int J Surg. 2018;56 (56):320-327. doi:10.1016/j.ijsu.2018.06.037

15. Takeda M, Sakuragi N, Okamoto K, et al. Preoperative serum SCC, CA125, and CA19-9 levels and lymph node status in squamous cell carcinoma of the uterine cervix. Acta Obstet Gynecol Scand. 2002;81 (5):451-457. doi:10.1034/j.1600-0412.2002.810513.x

16. Chen L, Zhang F, Sheng XG, Zhang SQ, Chen YT, Liu BW. Peripheral platelet/lymphocyte ratio predicts lymph node metastasis and acts as a superior prognostic factor for cervical cancer when combined with neutrophil: lymphocyte. Medicine (Baltimore). 2016;95(32):e4381. doi:10.1097/MD.0000000000004381

17. Simó M, Navarro X, Yuste VJ, Bruna J. Autonomic nervous system and cancer. Clin Auton Res. 2018;28(3):301-314. doi:10.1007/ s10286-018-0523-1

18. Bautista M, Krishnan A. The autonomic regulation of tumor growth and the missing links. Front Oncol. 2020;10:744. doi:10.3389/ fonc. 2020.00744

19. Gidron Y, Perry H, Glennie M. Does the vagus nerve inform the brain about preclinical tumours and modulate them? Lancet Oncol. 2005;6 (4):245-248. doi:10.1016/S1470-2045(05)70096-6

20. Arab C, Dias DP, Barbosa RT, et al. Heart rate variability measure in breast cancer patients and survivors: a systematic review. Psychoneuroendocrinology. 2016;68(68):57-68. doi:10.1016/j. psyneuen.2016.02.018

21. Zhou X, Ma Z, Zhang L, et al. Heart rate variability in the prediction of survival in patients with cancer: a systematic review and meta-analysis. J Psychosom Res. 2016;89(89):20-25. doi:10.1016/j. jpsychores.2016.08.004

22. Bijoor SN, Subbalakshmi NK, Banerjee S. Influence of cancer and its severity on vagal nerve activity assessed by time domain measures of heart rate variability. Res J Pharmacol Biol Chem Sci. 2016;7 (3):1215-1220.

23. Bettermann H, Kröz M, Girke M, Heckmann C. Heart rate dynamics and cardiorespiratory coordination in diabetic and breast cancer patients. Clin Physiol. 2001;21(4):411-420. doi:10.1046/j.13652281.2001.00342.x

24. Arab C, Vanderlei LCM, da Silva Paiva L, et al. Cardiac autonomic modulation impairments in advanced breast cancer patients. Clin Res Cardiol. 2018;107(10):924-936. doi:10.1007/s00392-018-1264-9

25. Hu S, Lou J, Zhang Y, Chen P. Low heart rate variability relates to the progression of gastric cancer. World J Surg Oncol. 2018;16(1):49. doi:10.1186/s12957-018-1348-z

26. Pan J, Tompkins WJ. A real-time QRS detection algorithm. IEEE Trans Biomed Eng. 1985;32(3):230-236. doi:10.1109/ TBME.1985.325532

27. Lipponen JA, Tarvainen MP. A robust algorithm for heart rate variability time series artefact correction using novel beat classification. J Med Eng Technol. 2019;43(3):173-181. doi:10.1080/ 03091902.2019.1640306

28. Task Force of the European Society of Cardiology and the North American Society of Pacing and Electrophysiology. Heart rate variability: standards of measurement, physiological interpretation and clinical use. Circulation. 1996;93(5):1043-1065. doi:10.1161/01. CIR.93.5.1043

29. Singh D, Vinod K, Saxena SC. Sampling frequency of the RR interval time series for spectral analysis of heart rate variability. $J$ Med Eng Technol. 2004;28(6):263-272. doi:10.1080/ 03091900410001662350
30. Welch P. The use of fast Fourier transform for the estimation of power spectra: a method based on time averaging over short, modified periodograms. IEEE Trans Audio Electroacoust. 1967;15:70-73. doi:10.1109/TAU.1967.1161901

31. Brennan M, Palaniswami M, Kamen P. Do existing measures of Poincaré plot geometry reflect nonlinear features of heart rate variability? IEEE Trans Biomed Eng. 2001;48(11):1342-1347. doi:10.1109/10.959330

32. Varon C, Lazaro J, Bolea J, et al. Unconstrained estimation of HRV indices after removing respiratory influences from heart rate. IEEE J Biomed Health Inform. 2019;23(6):2386-2397. doi:10.1109/JBHI.2018.2884644

33. Moody G, Mark R, Zoccola A, Mantero S. Derivation of respiratory signals from multi-lead ECGs. Comput Cardiol. 1985;12:113-116.

34. Tarvainen MP, Niskanen JP, Lipponen JA, Ranta-Aho PO, Karjalainen PA. Kubios HRV-heart rate variability analysis software. Comput Methods Programs Biomed. 2014;113(1):210-220. doi:10.1016/j.cmpb.2013.07.024

35. Sullivan GM, Feinn R. Using effect size-or why the $\mathrm{P}$ value is not enough. J Grad Med Educ. 2012;4(3):279-282. doi:10.4300/JGMED-12-00156.1

36. Yanaranop M, Sathapornteera N, Nakrangsee S. Risk factors of pelvic lymph node metastasis in cervical adenocarcinoma following radical hysterectomy and pelvic lymphadenectomy. J Med Assoc Thai. 2014;97(Suppl 11):S87-S95.

37. Fagotti A, Pedone Anchora L, Conte C, et al. Beyond sentinel node algorithm. Toward a more tailored surgery for cervical cancer patients. Cancer Med. 2016;5(8):1725-1730. doi:10.1002/cam4.722

38. Gulseren V, Kocaer M, Gungorduk O, et al. Preoperative predictors of pelvic and para-aortic lymph node metastases in cervical cancer. $J$ Cancer Res Ther. 2019;15(6):1231-1234. doi:10.4103/jcrt.JCRT_467_17

39. Zhou J, Ran J, He ZY, et al. Tailoring pelvic lymphadenectomy for patients with stage IA2, IB1, and IIA1 uterine cervical cancer. $J$ Cancer. 2015;6(4):377-381. doi:10.7150/jca.10968

40. Nanthamongkolkul K, Hanprasertpong J. Predictive factors of pelvic lymph node metastasis in early-stage cervical cancer. Oncol Res Treat. 2018;41(4):194-198. doi:10.1159/000485840

41. Brennan M, Palaniswami M, Kamen P. Poincaré plot interpretation using a physiological model of HRV based on a network of oscillators. Am J Physiol Heart Circ Physiol. 2002;283(5):H1873-H1886. doi:10.1152/ajpheart.00405.2000

42. Shaffer F, Ginsberg JP. An overview of heart rate variability metrics and norms. Front Public Health. 2017;5:258. doi:10.3389/ fpubh.2017.00258

43. McCraty R, Shaffer F. Heart rate variability: new perspectives on physiological mechanisms, assessment of self-regulatory capacity, and health risk. Glob Adv Health Med. 2015;4(1):46-61. doi:10.7453/gahmj.2014.073

44. Fadul N, Strasser F, Palmer JL, et al. The association between autonomic dysfunction and survival in male patients with advanced cancer: a preliminary report. J Pain Symptom Manage. 2010;39 (2):283-290. doi:10.1016/j.jpainsymman.2009.06.014

45. Wang YM, Wu HT, Huang EY, Kou YR, Hseu SS. Heart rate variability is associated with survival in patients with brain metastasis: a preliminary report. Biomed Res Int. 2013;2013:503421. doi:10.1155/2013/503421

46. Guo Y, Koshy S, Hui D, et al. Prognostic value of heart rate variability in patients with cancer. J Clin Neurophysiol. 2015;32 (6):516-520. doi:10.1097/WNP.0000000000000210

47. Cole SW, Nagaraja AS, Lutgendorf SK, Green PA, Sood AK. Sympathetic nervous system regulation of the tumour microenvironment. Nat Rev Cancer. 2015;15(9):563-572. doi: $10.1038 / \mathrm{nrc} 3978$

48. Pagani M, Lombardi F, Guzzetti S, et al. Power spectral analysis of heart rate and arterial pressure variabilities as a marker of sympatho-vagal interaction in man and conscious dog. Circ Res. 1986;59(2):178-193. doi:10.1161/01.RES.59.2.178 
49. Arai Y, Saul JP, Albrecht P, et al. Modulation of cardiac autonomic activity during and immediately after exercise. Am J Physiol. 1989;256(1 Pt 2):H132-H141. doi:10.1152/ajpheart.1989.256.1.H132

50. Alvarenga ME, Richards JC, Lambert G, Esler MD. Psychophysiological mechanisms in panic disorder: a correlative analysis of noradrenaline spillover, neuronal noradrenaline reuptake, power spectral analysis of heart rate variability, and psychological variables. Psychosom Med. 2006;68(1):8-16. doi:10.1097/01. psy.0000195872.00987.db

51. Moak JP, Goldstein DS, Eldadah BA, et al. Supine low-frequency power of heart rate variability reflects baroreflex function, not cardiac sympathetic innervation. Heart Rhythm. 2007;4(12):1523-1529. doi:10.1016/j.hrthm.2007.07.019

52. Goldstein DS, Bentho O, Park MY, Sharabi Y. Low-frequency power of heart rate variability is not a measure of cardiac sympathetic tone but may be a measure of modulation of cardiac autonomic outflows by baroreflexes. Exp Physiol. 2011;96(12):1255-1261. doi:10.1113/ expphysiol.2010.056259

53. de Lartigue G. Putative roles of neuropeptides in vagal afferent signaling. Physiol Behav. 2014;136(136):155-1569. doi:10.1016/j. physbeh.2014.03.011

54. Lin SC, Chen MF. Increased yin-deficient symptoms and aggravated autonomic nervous system function in patients with metastatic cancer. J Altern Complement Med. 2010;16(10):1059-1063. doi:10.1089/acm.2009.0487

55. Billman GE. The LF/HF ratio does not accurately measure cardiac sympatho-vagal balance. Front Physiol. 2013;4:26. doi:10.3389/ fphys.2013.00026

56. Hayes JD, Dinkova-Kostova AT, Tew KD. Oxidative stress in cancer. Cancer Cell. 2020;38(2):167-197. doi:10.1016/j.ccell.2020.06.001

57. Mantovani A, Allavena P, Sica A, Balkwill F. Cancer-related inflammation. Nature. 2008;454(7203):436-444. doi:10.1038/ nature 07205

58. Zahalka AH, Frenette PS. Nerves in cancer. Nat Rev Cancer. 2020;20 (3):143-157. doi:10.1038/s41568-019-0237-2

59. Erin N, Akdas Barkan G, Harms JF, Clawson GA. Vagotomy enhances experimental metastases of 4THMpc breast cancer cells and alters substance P level. Regul Pept. 2008;151(1-3):35-42. doi:10.1016/j.regpep.2008.03.012

60. Erin N, Duymuş O, Oztürk S, Demir N. Activation of vagus nerve by semapimod alters substance $\mathrm{P}$ levels and decreases breast cancer metastasis. Regul Pept. 2012;179(1-3):101-108. doi:10.1016/j. regpep.2012.08.001

61. Giese-Davis J, Wilhelm FH, Tamagawa R, et al. Higher vagal activity as related to survival in patients with advanced breast cancer: an analysis of autonomic dysregulation. Psychosom Med. 2015;77 (4):346-355. doi:10.1097/PSY.0000000000000167

62. Shi B, Wang L, Yan C, Chen D, Liu M, Li P. Nonlinear heart rate variability biomarkers for gastric cancer severity: a pilot study. $\mathrm{Sci}$ Rep. 2019;9(1):13833. doi:10.1038/s41598-019-50358-y

63. Ciurea AM, Gheonea DI, Schenker M, Mehedințeanu AM, Târtea GC, Vere CC. The prognostic correlation of heart rate variability at diagnosis with survival of patients with hepatocellular carcinoma. Diagnostics (Basel). 2021;11(5):890. doi:10.3390/ diagnostics 11050890

64. De Couck M, Gidron Y. Norms of vagal nerve activity, indexed by Heart Rate Variability, in cancer patients. Cancer Epidemiol. 2013;37 (5):737-741.

65. Gonzalez H, Hagerling C, Werb Z. Roles of the immune system in cancer: from tumor initiation to metastatic progression. Genes Dev. 2018;32(19-20):1267-1284.

66. Voss A, Schulz S, Schroeder R, Baumert M, Caminal P. Methods derived from nonlinear dynamics for analysing heart rate variability. Philos Trans a Math Phys Eng Sci. 2009;367(1887):277-296. doi:10.1098/rsta.2008.0232
67. Voss A, Kurths J, Kleiner HJ, et al. The application of methods of non-linear dynamics for the improved and predictive recognition of patients threatened by sudden cardiac death. Cardiovasc Res. 1996;31 (3):419-433. doi:10.1016/S0008-6363(96)00008-9

68. Huikuri HV, Perkiömäki JS, Maestri R, Pinna GD. Clinical impact of evaluation of cardiovascular control by novel methods of heart rate dynamics. Philos Trans A Math Phys Eng Sci. 2009;367 (1892):1223-1238. doi:10.1098/rsta.2008.0294

69. Ward-Hartstonge KA, Kemp RA. Regulatory T-cell heterogeneity and the cancer immune response. Clin Transl Immunol. 2017;6(9): e154. doi:10.1038/cti.2017.43

70. Tracey KJ. Reflex control of immunity. Nat Rev Immunol. 2009;9 (6):418-428. doi:10.1038/nri2566

71. Wang M, Zhao J, Zhang L, et al. Role of tumor microenvironment in tumorigenesis. J Cancer. 2017;8(5):761-773. doi:10.7150/jca.17648

72. Kessenbrock K, Plaks V, Werb Z. Matrix metalloproteinases: regulators of the tumor microenvironment. Cell. 2010;141(1):52-67. doi:10.1016/j.cell.2010.03.015

73. Qiao G, Chen M, Bucsek MJ, Repasky EA, Hylander BL. Adrenergic signaling: a targetable checkpoint limiting development of the antitumor immune response. Front Immunol. 2018;9:164. doi:10.3389/ fimmu.2018.00164

74. Huan HB, Wen XD, Chen XJ, et al. Sympathetic nervous system promotes hepatocarcinogenesis by modulating inflammation through activation of alpha1-adrenergic receptors of Kupffer cells. Brain Behav Immun. 2017;59:118-134. doi:10.1016/j.bbi.2016.08.016

75. Le CP, Nowell CJ, Kim-Fuchs C, et al. Chronic stress in mice remodels lymph vasculature to promote tumour cell dissemination. Nat Commun. 2016;7:10634. doi:10.1038/ncomms10634

76. Sood AK, Bhatty R, Kamat AA, et al. Stress hormone-mediated invasion of ovarian cancer cells. Clin Cancer Res. 2006;12 (2):369-375. doi:10.1158/1078-0432.CCR-05-1698

77. Karimi K, Bienenstock J, Wang L, Forsythe P. The vagus nerve modulates CD4+ T cell activity. Brain Behav Immun. 2010;24 (2):316-323. doi:10.1016/j.bbi.2009.10.016

78. Reijmen E, Vannucci L, De Couck M, De Grève J, Gidron Y. Therapeutic potential of the vagus nerve in cancer. Immunol Lett. 2018;202(202):38-43. doi:10.1016/j.imlet.2018.07.006

79. Tracey KJ. Physiology and immunology of the cholinergic antiinflammatory pathway. J Clin Invest. 2007;117(2):289-296. doi: $10.1172 / \mathrm{JCI} 30555$

80. Brown TE, Beightol LA, Koh J, Eckberg DL. Important influence of respiration on human $\mathrm{R}-\mathrm{R}$ interval power spectra is largely ignored. $J$ Appl Physiol. 1993;75(5):2310-2317. doi:10.1152/jappl.19 93.75.5.2310

81. Niederer D, Vogt L, Thiel C, et al. Exercise effects on HRV in cancer patients. Int J Sports Med. 2013;34(1):68-73. doi:10.1055/s-00321314816

82. Chuang CY, Han WR, Li PC, Young ST. Effects of music therapy on subjective sensations and heart rate variability in treated cancer survivors: a pilot study. Complement Ther Med. 2010;18 (5):224-226. doi:10.1016/j.ctim.2010.08.003

83. Hou L, Zhou W, Ren J, et al. Radiomics analysis of multiparametric MRI for the preoperative prediction of lymph node metastasis in cervical cancer. Front Oncol. 2020;10:1393. doi:10.3389/ fonc. 2020.01393

84. Calabrese A, Santucci D, Landi R, et al. Radiomics MRI for lymph node status prediction in breast cancer patients: the state of art. $J$ Cancer Res Clin Oncol. 2021;147(6):1587-1597. doi:10.1007/ s00432-021-03606-6

85. Huang YQ, Liang $\mathrm{CH}$, He L, et al. Development and validation of a radiomics nomogram for preoperative prediction of lymph node metastasis in colorectal cancer. $J$ Clin Oncol. 2016;34 (18):2157-2164. doi:10.1200/JCO.2015.65.9128 


\section{Publish your work in this journal}

Cancer Management and Research is an international, peer-reviewed open access journal focusing on cancer research and the optimal use of preventative and integrated treatment interventions to achieve improved outcomes, enhanced survival and quality of life for the cancer patient.

The manuscript management system is completely online and includes a very quick and fair peer-review system, which is all easy to use. Visit http://www.dovepress.com/testimonials.php to read real quotes from published authors.

Submit your manuscript here: https://www.dovepress.com/cancer-management-and-research-journal 